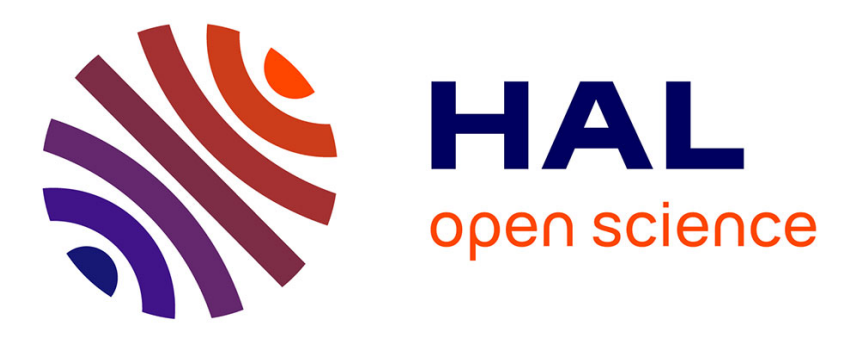

\title{
DNS Analysis of turbulent mixing in two-phase flows
}

Benjamin Duret, Gautier Luret, Julien Reveillon, Thibaut Ménard, Alain

Berlemont, François-Xavier Demoulin

\section{To cite this version:}

Benjamin Duret, Gautier Luret, Julien Reveillon, Thibaut Ménard, Alain Berlemont, et al.. DNS Analysis of turbulent mixing in two-phase flows. International Journal of Multiphase Flow, 2012, 40, pp.93-105. 10.1016/j.ijmultiphaseflow.2011.11.014 . hal-00649803

\section{HAL Id: hal-00649803 https://hal.science/hal-00649803}

Submitted on 8 Dec 2011

HAL is a multi-disciplinary open access archive for the deposit and dissemination of scientific research documents, whether they are published or not. The documents may come from teaching and research institutions in France or abroad, or from public or private research centers.
L'archive ouverte pluridisciplinaire HAL, est destinée au dépôt et à la diffusion de documents scientifiques de niveau recherche, publiés ou non, émanant des établissements d'enseignement et de recherche français ou étrangers, des laboratoires publics ou privés. 


\title{
DNS Analysis of turbulent mixing in two-phase flows
}

\author{
B. Duret, G. Luret, J. Reveillon, T. Menard, A. Berlemont and F.X. Demoulin* \\ UMR6614-CORIA, Technopôle du Madrillet, BP 12, Avenue de l'Université, 76801 Saint-Etienne-du-Rouvray \\ Cedex, France
}

\begin{abstract}
This study focuses on the evaporation and mixing process in turbulent two-phase flows with a direct resolution of the flow near the interface. A first approach, using a passive scalar to represent the evaporation and mixing process in a two-phase dense turbulent flow, has been developed and applied in a homogeneous isotropic turbulence over a large range of liquid volume fractions. This model is restricted to low vaporization rates, thus the interface is barely affected by the evaporation process. A statistical analysis of the vapor field is performed. Obtained results suggest that the beta PDF, frequently used in combustion modeling, are not adequate to represent the state of scalar mixing when interfaces are taken into account.

A spectral analysis of the velocity and the scalar field is carried out simultaneously in both phases as well as in each phase separately. A procedure using the liquid volume fraction field is employed to separate the contribution of each phase. The evaporation process does not affect the spectrum shape of the scalar, but it has a direct influence on the energy level of the scalar. Keywords: Evaporation, Two-phase flows, turbulent mixing, passive scalar, DNS, modeling, vaporization
\end{abstract}

*Corresponding author, demoulin@ coria.fr, Tel: +33232953 674, fax: +33232910485 


\section{Introduction}

The combustion of fuel issued from the evaporation of a liquid phase remains one of the major sources of energy, especially in the transport industry. Liquid injection in a combustion chamber is a critical step in fuel-air mixture preparation and its induced combustion. Hence, numerous studies have been devoted to the description and understanding of the injection process and its influence on vaporization and mixing. However, experiments are difficult, in particular in the vicinity of the dense zone of the spray. Numerical simulations could be of significant use if adapted methods are carefully developed following two main axes: the interface tracking ability and the phase change capture. These are sketched in Figure 1 along with several major liquid/gas interactions to be captured.

The last decade has seen the apparition of various numerical methods devoted to two-phase flow simulations with an accurate description of the interface position and evolution. Volume of Fluid (Hirt and Nichols (1981)), Level Set (Sussman et al. (1994)) and Front-tracking (Unverdi and Tryggvason (1992)) are the most common interface tracking methods developed to carry out direct numerical simulations of two-phase flows. In such simulations, discontinuities at the interface have to be treated carefully. With this aim, numerical methods such as the delta function method (Brackbill et al. (1992)) and the ghost fluid method (Fedkiw et al. (1999)) have been developed. The latter allows for applying sharp jump conditions at the interface and new works have proved it possible to accurately describe the primary atomization of liquid jets (Ménard et al. (2007), Desjardins et al. (2008), Shinjo and Umemura (2010)).

In the framework of combustion chamber modeling, interface tracking has to be coupled with an evaporation process. Such studies are relatively recent because of the complexity of multiple interactions between the various physical phenomena that prove to cover a wide range of different space and time scales (Tryggvason et al. (2010, 2005)). Solving phase change requires the resolution of the Navier-Stokes equations, but energy and species equations have to be considered with appropriate jump conditions at the interface. The interface velocity is also affected by the phase change, as are mass and energy transfers (Figure 1). All of these constraints lead to numerous problems, in particular the difficulty to estimate the local vaporization rate, which depends on 
the species and temperature gradients at the interface. Recently developed numerical methods to study these flows are expensive, and only basic configurations can be considered so far. One of the first tentative attempts was dedicated to bubbly flows with a two dimensional moving mesh Welch (1995). The mesh, which prevented wide deformations of the interface, limited this study. Moreover, computational costs were prohibitively expensive. Thereafter, Calimez (1998) made a major step forward by coupling phase change with the VOF method to study droplet group combustion in two-dimensions. Strong thermodynamic hypothesis were made to reduce computational cost and complexity. The same year, Juric and Tryggvason (1998) associated phase change with a front tracking method to simulate bubbly flows in two dimensions. But this method was unable, at the time, to manage significantly deformed interfaces. More recently, Tomar et al. (2005) studied phase change with a coupled Level Set VOF method but found it was necessary to smooth discontinuities across the interface. By using the Ghost Fluid method coupled with the Level Set method in two dimensional simulations, Tanguy et al. (2007) and Gibou et al. (2007) subsequently proposed a model to determine interface velocities aimed at solving continuity equations even including evaporation source terms. Strotos et al. (2008) analyzed droplet vaporization on a heated wall thanks to adaptive mesh refinement and the coupling between evaporation models and the VOF method. Schlottke and Weigand (2008) also developed a model to calculate velocities at the interface for deformed droplet vaporization in three dimensions with the VOF method.

All of the above mentioned methods are very promising. However, their complexity prevents them from being used in practical geometries. In this work, we propose to simplify the interactions between the various physical phenomena while focusing on the capture of an accurate vapor field. Saturation conditions at the interface are assumed to be constant, particularly the vapor pressure. No energy equation is resolved, and the liquid's vapor evolution is characterized by an inert scalar. Because the flow remains inert, the vapor mass fraction is equivalent to the mixture fraction, which defines the level of mixing between vapor and air. The saturation level of vapor in the domain can normalize this mixture fraction. It leads to the variable $Z$, which is thus bounded between 0 (no fuel vapor) and 1 (saturated fuel vapor). $Z$ is an inert scalar, the evolution of which is driven by a standard convection / diffusion equation in the gas phase. $Z$ is bounded by 1 at all interface positions. A sketch to illustrate the simplified evaporation process is shown in Figure 2. This 
is the first step towards a simultaneous understanding of atomization, vaporization and mixing processes in dense sprays. As a preliminary work, since the mixing characteristic time is very short compared to the evaporation delay, interface regression is not considered. We focus on the properties of $Z$ when the flow is shifting from very dense towards dispersed liquid presence, which mimics the liquid spatial evolution of an atomization process. To accurately control the various inputs of the simulation, a three-dimensional forced homogeneous turbulence of a gas / liquid flow is considered with a liquid volume fraction ranging from $1 \%$ to $99 \%$. Both phases will be resolved in DNS with the ARCHER code (Ménard et al. (2007)); the interface tracking method that has been used is a coupled Level Set/VOF method. A high-density ratio between the two phases is chosen to simulate realistic engine conditions. Both quantitative and qualitative aspects are analyzed.

In the following part of this work, the constitutive equations and numerical procedures are first described. The flow geometry is then depicted along with the various prescribed parameters. Next, a statistical analysis of the mixture fraction is carried out by studying the evolution of the probability density function of $Z$. Finally, an original spectral analysis is proposed, giving rise to the discussion of the links between droplet dispersion, the evaporation process and the final topology of vapor of liquid.

\section{Numerical considerations}

\subsection{Navier-Stokes and scalar equations}

The joint Level Set/VOF method is coupled with a projection method to carry out the direct numerical simulation of incompressible Navier-Stokes equations:

$$
\frac{\partial \boldsymbol{V}}{\partial t}+(\boldsymbol{V} . \nabla) \boldsymbol{V}=-\frac{\nabla p}{\rho}+\frac{1}{\rho} \nabla \cdot(2 \mu \boldsymbol{D})+\boldsymbol{g}+\boldsymbol{f}+\frac{1}{\rho} \sigma \kappa \delta(G) \boldsymbol{n}
$$

where $p$ is the fluid pressure, $\boldsymbol{V}$ the velocity vector, $\boldsymbol{g}$ the gravity vector, $\mu$ the dynamic viscosity, and $\boldsymbol{D}$ the viscous deformation tensor. At the interface, the surface tension force can be considered based on the Dirac function $\delta(G): \sigma$ is the surface tension, $\boldsymbol{n}$ the normal unit vector, $\kappa$ is the curvature computed from the Level Set function $G$ (definition of $\kappa, \boldsymbol{n}$ and $G$ are given in Section 2.2). The gravity term is neglected in this study. To solve the derivatives, a fifth-order scheme, 
WENO 5 (Shu (1997)), is used for convective terms, and a second-order central finite difference scheme is chosen for diffusive terms. (See Ménard et al. (2007) for further details concerning the numerical procedure.) A forcing method is necessary to maintain the turbulent kinetic energy at a prescribed level. This is achieved through the source term $f$, which induces linear forcing (Rosales and Meneveau (2005)). It results in $f=A v^{\prime}$ where $A$ is the forcing coefficient, $\boldsymbol{v}^{\text {' represents }}$ velocity fluctuations, and $\bar{v}$ is the mean flow velocity. In this paper, $\overline{(\cdot)}$ refers to volume averaging. A Reynolds decomposition has been applied to the velocity field $\boldsymbol{V}$. The evolution equation of the turbulent kinetic energy $k=\frac{1}{2} \overline{v^{2}}$ may be written:

$$
\frac{\partial k}{\partial t}+\nabla \cdot(\bar{v} k)=C_{k}+2 A k
$$

where $C_{k}$ regroups the typical energetic contributions (i.e. without forcing) for the sake of clarity. The estimation of $C_{k}$ involves a phase change and a liquid-gas interface. It is therefore complex to estimate $A$ directly from equation 2 , thus, the following two-stage procedure has been set up :

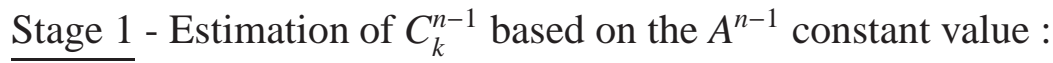

$$
C_{k}^{n-1}=\frac{k^{n}-k^{n-1}}{\Delta t}-2 A^{n-1} k^{n-1}
$$

Stage 2 - Computation of the forcing constant $A^{n}$ based on $C_{k}^{n-1}$ :

$$
2 A^{n} k^{n}=\frac{k_{c}-k^{n}}{\tau_{f}}-C_{k}^{n-1}
$$

where $k_{c}$ is the chosen level of kinetic energy, $\tau_{f}$ is a characteristic relaxation time $\left(\tau_{f}=3 \Delta t\right.$ has been retained in this study) to avoid a sharp forcing term. Thus, the force coefficient $A$ is computed at each time step to introduce the forcing term $f$ in the Navier-Stokes equations.

This method maintains a statistically stationary Homogeneous Isotropic Turbulence (HIT) in the whole domain.

To correctly describe the interface, the VOF method is used jointly with the Level Set to enforce mass conservation (Ménard et al. (2007)). For the sake of clarity, the mean value of the VOF method scalar is called the liquid volume fraction $\bar{\phi}$, and the local liquid volume fraction within a cell is $\phi$. They are defined as follows: $\bar{\phi}=V_{l} / V_{t}$, where $V_{l}$ is the total volume of liquid and $V_{t}$ 
the total volume of the domain. $\phi=V_{l c} / V_{c}$, where $V_{c}$ is the cell volume and $V_{l c}$ is the volume of liquid within a cell. $\phi$ respects the following transport equation:

$$
\frac{\partial \phi}{\partial t}+\boldsymbol{V} \cdot \nabla \phi=0
$$

The equation is discretized following the scheme as defined by Sussman and Puckett (2000); details on the coupled second-order conservative operator split advection are not restated here.

In this work, the value of the liquid volume fraction is of primary importance. Varying the liquid volume fraction $\bar{\phi}$ from 1 to $95 \%$ allowed our study to range from droplet flows to bubbly flows.

The choice of a passive scalar is representative of an evaporation process with a low temperature level at the interface, inducing a low saturation level of vapor at the interface. In such a case, assuming a local thermodynamics equilibrium at the liquid-gas interface, an evaluation of the saturation level of the vapor mass fraction $Y_{v s}$ can be made based on the Clausius-Clapeyron equation. The considered scalar $Z$ in this study is the normalized form of the vapor mass fraction: $Z=Y_{v} / Y_{v s}$. The scalar is thus equal to 1 at the interface; it then evolves in the gas phase due to convection and diffusion. In this preliminary study, it is considered that the amount of mass of liquid transferred in the gas phase is small enough if the saturation equilibrium is reached rapidly, which is the case. Therefore, the mass of liquid in the domain remains constant while the vapor phase evolution is considered and analyzed. This assumption leads to no significant phase change, but it is of practical interest to the study of mixing and scalar fields induced by vaporization when turbulence velocity fluctuations are greater than the Stephan flow velocity. The evolution equation of the inert scalar $Z$ may be written :

$$
\frac{\partial Z}{\partial t}+\nabla \cdot(V Z)=\nabla \cdot\left(D_{m} \nabla Z\right)
$$

where $D_{m}$ is the diffusion coefficient. In this paper, $D_{m}=v_{g}$ in the whole domain, which corresponds to a Schmidt number equal to unity. A fifth-order WENO scheme is used for the convective terms. A second-order central finite difference scheme is used for diffusive terms. The time integration is performed using a third-order Runge-Kutta scheme, except at the liquid-gas interface. A boundary condition is imposed at the interface in order to sustain the necessary value of $Z=1$ 
at the liquid-gas interface, allowing the resolution of equation 6 . The method is quite simple since the following relation

$$
\frac{\partial Z}{\partial t}=\frac{1-Z}{\tau_{w}}
$$

is resolved at the interface position. $\tau_{w}$ characterizes the time to reach the thermodynamic equilibrium in the mesh cell located at the interface. This status is assumed to always be satisfied at the interface. Moreover, the mesh cells are very fine (this is necessary to capture the interface curvature) thus $\tau_{w}$ must be very short while allowing a numerical tolerance. It has been set to $\tau_{w}=4 \Delta t$ in this study. Because the numerical methods of the solver are designed to cope with strong gradients (interface capture), $Z$ is set to 0 in the gas and 1 at the interface (and in the liquid) at the initial time of the simulation. The scalar equation is solved in the entire domain. In the liquid, $Z$ is fixed to unity during the simulation.

\subsection{Interface tracking method}

In the following, the interface tracking method and the treatment of discontinuities are recalled. More details can be found in the work of Ménard et al. (2007). Level Set methods use a continuous function to describe the interface (Sethian (1996), Osher and Fedkiw (2001)). This function is defined as the signed distance between any points of the domain and the interface. The 0 level curve of that function therefore provides the interface location. The convection equation to describe the motion of the interface in a given velocity field $\mathrm{V}$ reads:

$$
\frac{\partial G}{\partial t}+\boldsymbol{V} \cdot \nabla G=0
$$

Eq. (8) is the hyperbolic type, and the discretization method must combine a high convergence order and robustness. Thus, for the distance function field, a 5th-order WENO scheme (Shu (1997)) is used for convective terms. The normal unit vector $\boldsymbol{n}$ and the curvature of the interface $\kappa(G)$ are evaluated based on the Level Set function by :

$$
\boldsymbol{n}=\frac{\nabla G}{|\nabla G|}, \quad \kappa(G)=\nabla . \boldsymbol{n}
$$

The Level Set method G no longer remains a distance function when solving Eq. (8) because of the velocity gradients. A redistancing algorithm (Sussman et al. (1998)) is thus applied to 
keep $\mathrm{G}$ as the signed distance to the interface. A numerical resolution of Eq. (8) combined with the redistancing algorithm can induce mass loss in under-resolved regions. This loss is the main drawback of Level Set methods. To improve mass conservation, extensions of the method have been proposed (Sussman and Puckett (2000), Enright et al. (2002), Olsson and Kreiss (2005)). Specific studies have been developed to evaluate some of these methods (Ménard et al. (2007)). It has been found that the Coupled Level Set and Volume-of-Fluid method (CLSVOF, see Sussman and Puckett (2000)) is well adapted to capture atomization processes (Ménard et al. (2007), Lebas et al. (2009)). The main concept of this method is to benefit from the advantage of both Level Set and VOF strategies: mass loss is limited through the VOF method, and a fine description of interface properties is kept with the Level Set. The Level Set permits the reconstruction of an accurate interface in each cell and helps in the transport of the liquid volume fraction. The location of the interface is slightly modified to retain an amount of liquid in a computational cell that corresponds to the liquid volume fraction determined by the VOF approach. This displacement is used to ensure mass conservation.

Fluid dynamics equations are solved in the context of a low Mach number approach, based on a projection method for the direct numerical simulation of incompressible Navier-Stokes equations (detailed in Tanguy and Berlemont (2005)). The density and the viscosity depend on the sign of the Level Set function according to each phase (liquid and gas). To finalize the description of the twophase flow, jump conditions across the interface are taken into account with the Ghost Fluid (GF) method. In the GF method, ghost cells are defined on each side of the interface (Kang et al. (2000), Liu et al. (2000)). This prolongs each phase in order to allow smooth derivatives in the vicinity of the interface. As defined previously, the interface is characterized through the distance function, and jump conditions are extrapolated on a few nodes on each side of the interface. Further details on implementing the GF method to solve the Poisson equation with discontinuous coefficients can be found in the literature (Liu et al. (2000), Tanguy and Berlemont (2005)).

\section{Numerical configuration}

The solution is carried out in a 3D cubical domain with periodic boundaries similar to the study of Luret et al. (2010) and Luret et al. (2008). This configuration extends previous numerical 
studies on mixing in single-phase flows (Eswaran and Pope (1988b)) to two-phase flows with a fully resolved liquid-gas formulation.

\subsection{Parameters choice}

Solving realistic turbulent liquid-gas flows is difficult. Several limitations exist despite the significant increase of processor capacities. To define a configuration with effective interactions between the liquid-gas interface and the turbulence, the following dimensionless parameters have been retained: gaseous Weber number $W e_{g}=\rho_{g} \bar{k} L /(\sigma)=1$, liquid Weber number $W e_{l}=\rho_{l} \bar{k} L /(\sigma)=30$, liquid based Reynolds number $\operatorname{Re}_{l}=\sqrt{\bar{k}} L / v_{l}=310$, liquid Ohnesorge number $O h_{l}=\sqrt{W e_{l}} / R e_{l}=1.7710^{-2}$. Where $\bar{k}$ is the mean kinetic energy (usually equal to $k_{c}$ ), $\sigma$ the surface tension, $v$ the kinematic viscosity in the appropriate phase, $\rho$ the density and $L$ the box length. The grid resolution is $128^{3}$.

The corresponding set of fluid parameters is summarized in Table 1 .

\subsection{Validation}

Two issues have to be addressed concerning numerical validation in turbulent liquid-gas flows: First, numerical schemes used for single-phase flow DNS are not applicable for liquid-gas flows. Second, determining the smallest scales in turbulent liquid-gas flows remains an unsolved problem. Consequently, validating numerical configurations for turbulent liquid-gas flows can only be achieved partially. To overcome this difficulty, a systematic procedure has been defined. The first step in this procedure involves validating the numerical method used for liquid-gas flows in a single-phase flow configuration by comparing it to classical non-dissipative DNS code. Then, the same numerical parameters are kept to carry out numerical simulations in two-phase flows. Finally, for one liquid-gas flow configuration, the mesh resolution is doubled in all directions to study the effect of the small scales that are not captured with the lower resolution.

\subsubsection{Single Phase flows}

Numerical schemes are typically non dissipative and accurate in DNS of single phase flows. It is possible to compute converged results even for fifth-order moments by using spectral methods 
or high order difference schemes. The estimation of the Kolmogorov scale is wellknown in a HIT configuration, and the mesh size must be of the same order or slightly smaller than the Kolmogorov scale $\left(1.5 \geqslant \frac{\eta}{d x} \geqslant 1\right.$, see Eswaran and Pope (1988a)).

This kind of numerical scheme is inappropriate in the context of two-phase flows, particularly when dealing with high-density gradients. In the ARCHER code for instance, a fifth-order WENO scheme (Shu (1997)) is used as a good compromise between accuracy and robustness. A counterpart of its robustness is the possible introduction of numerical dissipative effects, which can affect small-scale turbulence. A comparison between the ARCHER code and a validated DNS code (used for single-phase flows in many configurations (ASPHODELE, Guichard et al. (2004), Reveillon and Vervisch (2005), Reveillon and Demoulin (2007) and Chaisemartin et al. (2009)) has been performed to evaluate this effect. An analysis of two key quantities (turbulent kinetic energy and dissipation) reveals that both codes give similar results for the parameters and the configuration presented in this paper in the case of single-phase flows. This indicates that the single-phase flow case is sufficiently resolved with the ARCHER code for the proposed set of parameters.

Additionally, to validate the forced HIT, an estimation of the accuracy of the forcing method has been performed for the configuration described by Rosales and Meneveau (2005), where results have been obtained with spectral methods and high-order central difference schemes. The coefficient $A$ of the forcing scheme (Eq. 2) is set to the same value as the one used by Rosales and Meneveau (2005). The resulting turbulent kinetic energy found by Rosales and Meneveau (2005) is well recovered with the ARCHER code. This result confirms the ability of the ARCHER code to accurately reproduce the balance between turbulence production (forcing scheme) and dissipation.

\subsubsection{Two-phase flows}

Determining whether the resolution is high enough is more complex in two-phase flow configurations. Since a definition equivalent to the Kolmogorov scale to determine the smallest scale of the flow is not yet available, it is impossible to estimate the necessary resolution. The smallest scales remain unknown; they are not fully resolved even with the highest resolved DNS (Shinjo and Umemura (2010)). Hence, we have chosen to set the resolution for two-phase flows equal to the resolution determined for single-phase flow cases. 
However, as a complement, a grid convergence is conducted to validate behavior of the flow studied in this article. Figure 3 shows velocity spectra of a $128^{3}$ configuration and a $256^{3}$ case (for $\bar{\phi}=5 \%$ and same box length).

Some energy has been transferred to small scales in comparison with two-phase flow cases. This effect is dumped by the WENO 5 scheme in the $128^{3}$ configuration. However, a large range of the spectra remains unchanged, allowing us to consider that only small scales are not well captured. The $256^{3}$ case is shown in the appropriate figures when possible, illustrating that the main results of this paper are not significantly affected by increasing the resolution.

\subsection{Initializing phase}

The periodic box initialization is based on the work of Luret et al. (2008, 2010). Eight droplets are introduced in each corner of the domain; the sum of their volume is equal to the fixed liquid volume fraction. All droplets have an initial rotational velocity in accordance with the prescribed kinetic energy. Linear forcing is inactive at the beginning of the simulation. The flow is quickly destabilized. Next, the linear forcing is activated and the turbulent kinetic energy reaches its desired level with a statistically stationary state. Then, the vaporization process is switched on. The normalized scalar $Z$ is used to study the mixing of vapor with the ambient air in the gas phase. The initial scalar field is set to $Z=1$ at the interface, $Z=1$ in the liquid for continuity reasons, and $Z=0$ in the gas.

\section{Discussion}

The scalar is analyzed from both statistical and spectral points of view. The first objective is to provide statistical information about the scalar field and the vaporization process. For combustion modeling, which is the final outcome of the atomization in a chamber, the statistical distribution of the vapor field is of primary importance. Previous works have been carried out either to characterize it with DNS of dispersed phase flows (Reveillon and Vervisch (2000)) or to propose models (Demoulin and Borghi (2002)). However, our study presents the first analysis involving a complete DNS of the fully resolved vapor field from the interface up to the far field of the spray. 


\subsection{Liquid/gas flow}

Color levels of the instantaneous scalar field $Z$ are presented in Figure 4 for six values of $\bar{\phi}$ ranging from $1 \%$ and $95 \%$. The liquid/gas interface is also shown thanks to a 3D surface representation. At this point, configurations with a small liquid volume fraction (1\% and $5 \%$ ) are considered as dispersed cases when compared with dense configurations that are greater than $90 \%$. This assumption has to be relativized to the common definition of dispersed flows, which considers volume fractions generally below $0.001 \%$. These configurations cannot be represented by interfacial flows, and gaseous DNS solvers associated with Dispersed Phase Solvers (DPS) are commonly used in this case (Reveillon and Demoulin (2007)). This means that the most dispersed cases studied in this paper (based on interface transport) may be considered as dense from the DNS-DPS point of view.

At low $\bar{\phi},(1 \%$ and $5 \%)$, small liquid structures with almost spherical shapes are present in the domain. These droplets are not affected by the surrounding gas because of the high-density ratio between the two phases. However, the forced turbulence causes the liquid packets to collide regularly. Small gas structures appear (bubbles) for a high liquid volume fraction (95\%). They are embedded in a significant quantity of liquid. This case may be called "dispersed bubble flow". The gas phase is strongly affected by the surrounding liquid flow, and bubbles are stretched by the shear force due to the liquid phase. The intermediate case $(\bar{\phi}=50 \%)$ is more complex. Both phases are present in similar proportions. The gas follows mainly the liquid motion, but collision and coalescence are less commonly observed.

Despite a relatively small Weber number value, the interface is very wrinkled in all presented cases. Since accurately resolving the interface is of crucial importance to capture breakups and vaporization rates, the usual vaporization models based on spherical droplets are not relevant. In dense cases, the gas phase volume is smaller and scalar gradients are then higher, leading to quick vaporization and mixing. The gas reaches the saturated state after a characteristic time $\tau_{v p}$. This time span can be estimated from the temporal evolution of the mean scalar value, presented in Figure 5. $t^{*}$ is the time normalized by a characteristic turbulent time $\tau_{\text {turb }}=L / u^{\prime}$ based on the velocity root mean square $u^{\prime}$ and the box length $L$. Dense liquid flows $(\bar{\phi}=95 \%)$ reach the saturation level $\left(\tau_{v p}=0.38 t^{*}\right)$ very quickly while a comparatively long delay $\left(\tau_{v p}=10.2 t^{*}\right)$ is 
necessary for the dispersed configuration $\bar{\phi}=5 \%$. It defines the time range necessary for a flow with any intermediary liquid volume fraction to reach the saturation. For example, when $\bar{\phi}=50 \%$, then $\tau_{v p}=1.97 t^{*}$. Thus, $\tau_{v p}$ is correlated to $\bar{\phi}$ but in a strongly non-linear way. The high-resolution case presents a similar evolution of the mean value of the scalar $\bar{Z}$. A small discrepancy can be noticed showing a weak effect of small scales captured by the higher mesh resolution. However, the differences noticed for each level of liquid concentration are much greater.

\subsubsection{Mixture fraction fluctuations}

To characterize scalar mixing and the evaporation process, the scalar RMS $Z^{\prime}=\sqrt{\overline{(Z-\bar{Z})^{2}}}$ is computed in the gas phase at each time step. The theoretical maximum value of $Z^{\prime}$ is given by $Z_{m}^{\prime}=\sqrt{\bar{Z}(1-\bar{Z})}$. This case represents a field of scalar $Z$ with a maximum of fluctuations. Both $Z^{\prime}$ and $Z_{m}^{\prime}$ have been plotted in Figure 6 for three different liquid volume fractions. An effect of the mesh resolution on $Z_{m}^{\prime}$ can be observed, which is simply an amplification of the difference observed in Figure 5 for $\bar{Z}$. On the contrary, the difference is attenuated as far as the RMS $Z^{\prime}$ is concerned, showing a small effect of mesh resolution on the result.

A comparison with the results found by Eswaran and Pope (1988b) and Reveillon and Vervisch (2000) can be carried out. The study of Eswaran and Pope concerned turbulent mixing in purely gaseous flows. Therefore, no source of mixture fraction fluctuations exists. Thus, an exponential decay of $Z^{\prime}$ is observed because of scalar dissipation, which is the sole phenomenon acting on the mixture. The study of Reveillon and Vervisch (2000) concerned turbulent mixing in very dispersed two-phase flows. Droplets were considered as a point source of fuel vapor. The authors showed that the scalar RMS increases initially because of the vaporization term $\dot{Z}_{w}$ (which generates local fluctuations of vapor) and then decreases due to the scalar dissipation rate. In fact, dissipation overcomes the production of fluctuations by vaporization after a specific characteristic time. For interface tracking DNS, a similar behavior of $Z^{\prime}$ is observed in Figure 6. However, contrary to the study of Reveillon and Vervisch (2000), the mixture fraction increase is not only due to the apparition of a pocket of vapor around a group of droplets but also due to the characterization of the vapor boundary layer around each droplet or liquid structure. We have therefore made progress in the mixing description. This allows us to consider high liquid volume fractions in the study. As 
shown in Figure 6, the higher the liquid volume fraction, the higher the max of the RMS. This can be explained by the reduction of the gas volume available for mixing, then by a decrease of the vaporization delay, which is shorter when $\bar{\phi}$ increases. Thus, the balance between dissipation and production is modified, causing higher fluctuations of the mixture fraction.

An interesting point can be made by not only observing the first two moments of the mixture fraction but also its complete statistical evolution through the evolution of its probability density function (PDF).

\subsubsection{PDF evolution}

Inert scalar PDF (denoted $f(Z)$ ) are of major importance when studying turbulent inert or reactive flows. In particular, numerous combustion models are based on the PDF of $Z$ because it allows us to compute the filtered or averaged chemical source term through $\overline{\dot{\omega}}_{c}=\int_{0}^{1} \dot{\omega}_{c}\left(Z^{*}\right) f\left(Z^{*}\right) d Z^{*}$. It is generally possible to analytically reconstruct the shape of the PDF that is required to close the reaction terms based on our knowledge of the first two moments (mean and variance) of the mixture fraction (Borghi (1988)). The prescribed analytical PDF shape required to describe statistical properties of $Z$ must be able to evolve from a double delta function (high air/vapor segregation level) to a single delta function centered on the mean when perfect mixing is reached. A mathematical function that allows this kind of behavior is the beta function (see Libby and Williams (1994)), which is based on an expression that uses the first two moments of $Z$ as entry parameters: the mean $\bar{Z}$ and the variance $\overline{Z^{\prime 2}}$. It gives :

$$
f(Z)=Z^{a-1}(1-Z)^{b-1} \frac{\Gamma(a) \Gamma(b)}{\Gamma(a+b)}
$$

where $\Gamma$ is the gamma function. The two parameters $a$ and $b$ are related to the mean and variance through

$$
a=\bar{Z} c, b=(1-\bar{Z}) c \text { with } c=\frac{\bar{Z}(1-\bar{Z})}{\overline{Z^{\prime 2}}}-1
$$

DNS of dispersed flows does not resolve the flow at the droplet length scale since they are considered as point sources of mass, momentum and energy. This implies filtering at the interface 
level. Thus, the considered numerical system leads to a small numerical dispersion that can affect the small scale mixing analysis. It appears that the beta function was verified to capture the turbulent mixing in many experimental and numerical works in single-phase (Eswaran and Pope (1988b)) or multiphase flows (Reveillon and Demoulin (2007); Libby and Williams (1994)) in the framework of dispersed droplets. However, this point should be verified when the entire scalar field is resolved.

To confirm this point, the flow is entirely resolved in both phases in this study, including the mass boundary layers located at the interface. Therefore, present results can be used to verify if beta functions are still valid when the vapor field is solved at the interface vicinity whatever the liquid volume fraction. However, we are particularly interested in results at a low liquid volume fraction, which may be used to evaluate the usual dispersed DNS mixing results. Nevertheless, there is a limitation. As stated in the introduction, the numerical cost can be exceedingly high when completely solving both phases at the interface, and the more the spray is dispersed the more expensive simulations are. Consequently, the most dispersed case presented here corresponds to a liquid volume fraction of $1 \%$. More intensive computations are necessary to decrease this liquid volume fraction towards a smaller value, but this will be the purpose of further study. In this paper, we are focusing on the transition from dense flows ( $\bar{\phi}=99 \%)$ to relatively dispersed flows $(\bar{\phi}=1 \%)$.

To compare exact PDF evolutions observed in interface tracking DNS with the beta PDF, the evaporation process is observed at five fixed moments, represented in Figure 7. Each moment corresponds to a specific mean value of $Z: \overline{Z_{1}}=0.1, \overline{Z_{2}}=0.3, \overline{Z_{3}}=0.5, \overline{Z_{4}}=0.75$ and $\overline{Z_{5}}=0.95$, in order to study vaporization from the initialization $\left(\overline{Z_{1}}\right)$ to the saturation state $\left(\overline{Z_{5}}\right)$. The following instants: $t_{\bar{z}_{1}}^{*}, t_{\bar{z}_{2}}^{*}, t_{\bar{z}_{3}}^{*}, t_{\bar{z}_{4}}^{*}, t_{\bar{z}_{5}}^{*}$ represent the delays necessary to reach each step. These delays differ depending on the initial liquid volume fraction $\bar{\phi}$ that is considered. In the description of the results, the scalar PDF $f(Z)$ is plotted at these five considered moments. This method to study the scalar statistically is used for all liquid volume fractions.

Comparisons of effective scalar PDF obtained from DNS (DNS-PDF) and beta-presumed PDF 
$(\beta-\mathrm{PDF})$ corresponding to similar moments are shown in Figure 8 with the exception of PDF concerning high liquid volume fractions $(\phi=90,95 \%)$, in which case statistics are not determined because of the lack of samples since only few bubbles are present in the flow.

DNS-PDF of the most dispersed cases (up to $\bar{\phi}=10 \%$, Figure 8 - solid lines) at first present a peak corresponding to $Z=0$ statistics (the initial gaseous state in the simulations). Then evaporation maintains a $Z=1$ level at the interface, and mixing occurs with the joint effects of convection and diffusion. This mixing leads to a widening of the peak whose maximum value decreases at first. Because evaporation is still in progress, the mean mixture fraction level increases towards its maximum value 1, corresponding to a saturated state in the liquid. As $Z$ increases towards 1 , the maximum of the DNS-PDF begins to increase again when the mixture is at approximately a third of the saturation level. Dispersion is decreasingly effective, and the PDF tends toward a Dirac function. In the case of a liquid volume fraction equal to $50 \%$ we see a different behavior since the initial statistical peak at $Z=0$ shows a very large dispersion of the statistics, and a large variety of mixture fraction levels can be observed.

If the mean and the variance of the mixture fraction are used to reconstruct the PDF based on a beta function, it is apparent (Figure 8 - dashed lines) that the $\beta$-PDF is not able to capture the mixing properties. The mixture fraction is considerably spread around the mean, and several orders of magnitude of the statistical level can be observed. A counter intuitive observation can even be made since the $\beta$-PDF closely matches with the DNS-DPS when a dense flow is considered $(\bar{\phi}=50 \%)$. But the more the flow is dispersed, the farther from reality the $\beta$-PDF is located. We could have supposed an inverse relationship since the dispersed case is controlled by the gaseous mixing. Moreover, different results are observed when using a DNS-DPS solver in the framework of a much more dispersed flow (Reveillon and Demoulin (2007)). Droplet sizes are assumed to be smaller than the Kolmogorov length scale in DNS-DPS. Consequently, they are also smaller than the mesh size. Local vaporization source terms (computed with evaporation models) are distributed on neighboring mesh nodes. The flow around and inside the droplet is not resolved, neither are boundary layers. We completely solve the flow around the droplet, including boundary layers, with interface tracking DNS. Additionally, since present two-phase flows are less dispersed, the volume occupied by scalar boundary layers over the total volume of gas available is greater 
than in dispersed DNS. These two facts induce the presence of specific statistical information that can be captured only with interface tracking solvers: the saturated interface leads to the permanent presence of unitary values of the mixture fraction $Z$. Because of a scale effect, this is almost invisible on the DNS-PDF curves plotted in Figure 8. A slight peak at $Z=1$ can be observed for all curves of the case $\bar{\phi}=10 \%$, for example. It seems very small compared to the main peak centered close to the mean value of $Z$. However, this peak is the cause of the $\beta$-PDF failure to represent turbulent mixing. The $\beta$-PDF's flattening is due to its inability to capture information at the saturated level. An example has been drawn in Figure 9, representing two PDF with exactly the same mean and variances. The solid line curve presents two singularities: first a classical Gaussian shape curve and then a Delta function centered in $Z=1$. The presence of this secondary peak, which corresponds to the saturated area in the boundary layers of our DNS simulations, leads to unbalanced statistics. If the $\beta$-PDF corresponding to the solid line PDF is plotted (dashed curve), it becomes evident that it is not able to cope with the presence of the interface without a specific treatment.

\subsubsection{PDF shapes}

In Figure 10, normalized shapes of the DNS-PDF $\left(f(z) \times Z^{\prime}\right.$ with respect to $\left.(Z-\bar{Z}) / Z^{\prime}\right)$ are plotted for each part of the vaporization process. They reveal an important asymmetry. First, the vaporization process occurring for high values of $\mathrm{Z}$ affects the queue of the PDF. Even if the peak is almost invisible because of scale effects, it has been proven above to have a prevalent impact on $Z$ statistics. As a consequence, when studying dispersed cases $(\bar{\phi}<10 \%)$, the peak is logically located slightly below the scalar mean value with a dispersion that is the result of the mixing process. Previous studies of purely gaseous flows (Eswaran and Pope (1988b)) have shown that mixing leads to a stationary PDF shape, close to a Gaussian shape, with a probability peak located at the mean value.

In our case, PDF tend to be self-similar over time for dispersed cases $(\bar{\phi}=1,5,10 \%)$, except during the first stage (P1 in Figure 10) of the vaporization process. This first stage is the direct result of the interface topology at the beginning of the vaporization. Then, turbulent mixing far 
from the boundary layer takes over, which leads to a self-similar shape of the PDF. However, if the liquid volume fraction increases, the boundary layers strongly impact the PDF, and no selfsimilarity can be observed.

Note also that the Gaussian shape observed in the framework of purely gaseous flows is not retrieved in the framework of two-phase flows. Instead, a PDF with a shape close to a lognormal function may be seen in Figure 10. This is an important result for future model development. Even if a correction is proposed, which is necessary to take the presence of an interface into account, the $\beta$-PDF would not be able to retrieve the desired shape, and a lognormal shape appears to be more appropriate.

This assumption is confirmed by the fact that the shapes tend to be self-similar over time for dispersed cases $(\bar{\phi}=1,5,10 \%)$, except during the first stage of the evaporation process. A mathematical function of this shape could replace the beta-function in this particular case.

This property is lost in intermediate and dense cases. Nevertheless, similar shapes are observed for part two and three of the evaporation process: two Dirac functions at $Z=0$ and $Z=1$ may be observed. For part four and five a spread shape over $\mathrm{Z}$ values is found with a high probability $(Z=1)$. In these cases, the vaporization process is faster than the scalar mixing thus the PDF do not have the time to converge toward a common shape; they are more dependent on interface geometry.

\subsection{Energy spectra analysis}

\subsubsection{Velocity fields}

Two-phase spectra. Spectra of the velocity fluctuations of the gas/liquid mixture are presented in Figure 11. Spectra have a similar shape whatever the liquid volume fraction is. This shape is very close to those observed in single-phase flows, which is compatible with the fact that the liquid volume fraction level has no visible impact. This is an important first observation. However, this subject is not discussed in this work since a forcing procedure is used to maintain a constant turbulent kinetic energy by adding a term in the momentum equation. This term could be at the origin of the spectra similarity. To clarify this point, a different configuration with a natural production 
of turbulent kinetic energy, like a shear flow, has to be considered. Another stumbling block lies in the lack of development of analysis procedures of two-phase flows with a high level of liquid volume fraction and a high liquid / gas density ratio. The impact of this ratio on the turbulence spectra definition and evolution is still uncertain. Spectra of velocity fluctuations, liquid volume fractions, and vapor scalars for interfacial flows with strong deformations of the interface (including high density ratios) have never been studied prior to this work, which presents some preliminary reflections on the subject. However, this is a major topic with a wide range of applications and issues. As a preliminary procedure, a common spectral analysis (generally applied in one-phase flows) has been carried out since there is no velocity jump at the interface position. Thus, a FFT procedure on the periodic domain is carried out to extract non-dimensional spectral density of energy visible in Figure 11. However, a phase separation procedure is necessary to discriminate both liquid and gas spectra.

Phases separation. FFT are applied to the entire domain, and the considered signal must be defined on all grid points, which complicates the analysis of phase-separated variables. Therefore, it is necessary to find a method to isolate each phase from a spectral point of view. If the velocity fluctuations are considered, the liquid volume fraction is used to separate liquid and gas in the physical space prior to FFT. Accordingly, it is possible to define

$$
\boldsymbol{V}_{g}=\left(\boldsymbol{V}-\overline{\boldsymbol{V}_{g}}\right)(1-\phi)
$$

to study the gas phase and

$$
\boldsymbol{V}^{\prime \prime}=\left(\boldsymbol{V}-\overline{\boldsymbol{V}_{l}}\right) \phi
$$

for the liquid phase. $\boldsymbol{V}_{l / g}$ " is the velocity fluctuation's vector of the liquid $(l)$ or gas $(g)$ phase, respectively. $\overline{\boldsymbol{V}}_{l / g}$ is the mean velocity vector computed exclusively in the considered phase. Fluctuations of the variable in the complementary phase are nullified when a given phase is studied by using this procedure.

First, it is important to quantify the effect of nullifying velocity fluctuations by using the local liquid volume fraction $\phi . \phi$ is a bimodal variable. It may be equal to either 0 or 1 . Its maximum 
RMS may be determined thanks to $\phi_{m}^{\prime}=\sqrt{\bar{\phi}(1-\bar{\phi})}$. Therefore, the intermediate case corresponding to a liquid volume fraction $\bar{\phi}=50 \%$ undergoes the strongest fluctuations. Then, a symmetry around this maximum value is respected, and a dispersed droplets case $(\bar{\phi}=5 \%)$ presents a similar maximum RMS of liquid volume fraction to a dispersed bubble case $(\bar{\phi}=95 \%)$. A spectral analysis of $\phi$ is performed in Figure 12, and this hierarchy is also observed between spectra. They have been plotted for various liquid volume fractions. The most energetic case corresponds to $\bar{\phi}=50 \%$. All spectra present an inertial range with a $-5 / 2$ slope in the log-log representation. Considering the nature of the liquid volume fraction variable, no dissipation range is observed in the high frequency region. This spectrum shape influences the phase conditioned velocity fields since they are defined as a product of the velocity with this scalar $\phi$. This point has to be considered in the following.

Phase separated velocity spectra. At this point, it is possible to consider the phase separated velocity spectra, which have been plotted in Figure 13. All spectra have been normalized by the corresponding kinetic energy and focus is given to the various shapes that may be observed. Two major observations can be made concerning, on the one hand, the position of the maximum of the energy for each phase and, on the other hand, the inertial range.

First, let us consider the case $\bar{\phi}=50 \%$ where the same volume of liquid and gas are evolving in the domain. Spectra have exactly the same shape except for a slight difference in the high frequency range. If the configurations with droplets are considered (low liquid volume fraction: $1 \%, 5 \%$ and $10 \%$ ), there is a shift of the maximum of energy, which is then separated for both phases: the gas phase presents a maximum of energy for the lowest frequency range (i.e. for the largest length scales) contrary to the liquid phase. This shift corresponds to the droplet structures that are smaller than the largest gaseous vortices. Moreover, there is a discrepancy between inertial ranges of both liquid and gas phases that do not have similar slopes. If configurations with bubbles are examined (high liquid volume fraction 90\%, 95\%), the maximum of energy, occurring at the low frequency range, is that of the liquid phase. The small size of the bubbles compared to the turbulent liquid vortices leads to a position of the maximum of the gas energy at a higher frequency. 
If two symmetrical dispersed cases are considered, such as $\bar{\phi}=5 \%$ (droplets) and $\bar{\phi}=95 \%$ (bubbles), both phases interact very differently because of the significant difference of density between gas and liquid. Some bubbles in a liquid flow strongly undergo the liquid evolution and structures motion. Yet, droplets in a gaseous flow possess a strong inertia (in the cases considered in this work). Their motion will be weakly modified by the small structures of the flows and droplet/droplets interactions have a strong impact on their trajectory. Consequently, there is no real symmetry in the evolution of both phases when bubble and droplet dispersed cases are compared. The characteristic length scales are different as well. To serve as an example, an estimation of the equivalent Mean Sauter Diameter (SMD) $L_{D 32}$ of a mono-disperse spray corresponding to the same interface density area present in the DNS case is calculated $\left(L_{D 32}=6 \bar{\phi} / \Sigma\right.$ for droplet flows and $L_{D 32}=6(1-\bar{\phi}) / \Sigma$ for bubble flows, see Lebas et al. (2009) for details). As a reference and to demonstrate the non-symmetrical evolution of both phases the droplet SMD is equal to $29.82 \mu m$ in the case $\bar{\phi}=10 \%$, whereas in the bubble dispersed case $(\bar{\phi}=90 \%)$ the bubble SMD is equal to $21.97 \mu \mathrm{m}$. This estimation is commonly used in the Euler/Lagrange formalism to characterize the transition between the Lagrangian particles (secondary atomization) and the Eulerian description (primary atomization).

The characteristic length scale of the liquid volume fraction spectrum $Ł_{\sigma}$ has been plotted in Figure 13 to compare its value to the maximum value obtained from the velocity spectrum. The more one phase is dispersed, the more its characteristic length scale becomes equivalent to the liquid volume fraction length scale. This may be a bias of the method used to distinguish between each phase velocity spectrum that uses the liquid volume fraction, but it could also be an effect of the surface tension that creates droplets (bubbles). These droplets (bubbles) enclose a pocket of liquid (gas) that evolves mainly at the same velocity, creating a scale of velocity fluctuation related to the liquid volume fraction length scale.

\subsubsection{Scalar fluctuations}

A clear definition of the scalar spectrum has to be given prior to any analysis. The mixture fraction $Z$ is defined only in the gas phase, but we need to provide $Z$ information in the liquid area of the flow without corrupting the resulting spectrum. Three basic approaches can be considered to 
separate both phases in the framework of the mixture fraction analysis: nullify scalar fluctuations by prescribing the $Z$ values to its mean in the liquid or force $Z$ values to either 0 or 1 in the liquid phase. The objective is to study $Z$ fluctuations, thus the last two solutions are prohibited since artificial fluctuations would be added. These fluctuations would follow those of the phase function $\phi$, which would corrupt the results. The first approach seems to be a good compromise. $Z$ values along a given profile are shown in Figure 14 for a better understanding of the modified scalar field. Affecting the mean of $Z$ in the liquid area will not affect the spectral analysis of the mixture fraction fluctuations.

It is apparent that the most important part of the vapor scalar field remains unaffected by the boundary layers. In this case, scalar mixing should remain dominated by single-phase mixing. This is possibly the reason for recovering a $-5 / 3$ slope in the spectrum.

A temporal evolution of scalar spectra is shown in Figure 15 for short times $\left(t^{*} \epsilon\left[t_{0}^{*}, t_{\bar{z}_{1}}^{*}\right]\right)$. Initially, the scalar spectra have a particular shape with a uniform repartition of the kinetic energy on the different scales and quickly tend to a stationary spectrum with a $k^{-5 / 3}$ inertial range. The effect of the turbulence appears to be prevalent in comparison to the evaporation process. When looking at Figure 16, which plots the temporal evolution of scalar spectra at times $t_{\bar{z}_{1}}^{*}, t_{\bar{z}_{2}}^{*}, t_{\bar{z}_{3}}^{*}, t_{\bar{z}_{4}}^{*}$, $t_{\bar{z}_{5}}^{*}$, the same shape is retrieved.

The scalar seems to have an inertial range larger than the velocity spectra. This fact has been duplicated in single-phase flows (Danaila and Antonia (2009)). We recall that the liquid volume fraction can also affect the shape. The energy level decreases as time increases. Because scalar values tend to the scalar mean as time increases (as we can see on the PDF shown in Section 4.1), scalar fluctuations are reduced until the saturation state is reached (corresponding to $Z=1$ in the entire domain).

The preceding section reveals the difficulty in studying the scalar spectrally in a two-phase flow configuration. Our first result shows that it is possible to recover single-phase flow behavior in the dispersed limit. For a higher liquid volume fraction, the influence of the complementary phase spectrum hides the vapor scalar behavior. 


\section{Conclusion}

This paper presents the first attempt to study mixing under the constraint of a turbulent liquidgas flow and evaporation with full resolution of both phases. It is evident that further works are needed to fully explore such complex phenomena. Among them, the effect of forced turbulence, mesh resolution, and the methods used to analyze each phase separately are of primary interest. However, by comparison to previous works that used a discrete approach for the minority phase, the present work gives valuable indications for modeling purposes.

DNS have been performed in homogeneous isotropic turbulence with a full resolution of both phases. Original results have been found when studying the scalar from both statistical and spectral points of view. Exact PDF shapes obtained with DNS are much closer to a lognormal shape than the commonly presumed beta PDF. Moreover, the interface mass boundary layer introduces a statistical flaw that is not captured by any presumed PDF. This is an important result since presumed beta PDF are often used in combustion modeling to describe the equivalence ratio field and to close the chemical source term.

Spectral analysis has been conducted for the first time in such turbulent liquid-gas and interfacial flows. A method has been proposed to isolate each phase in the spectral domain based on the local liquid volume fraction. The majority phase spectrum corresponds to the total velocity, except at a high frequency range, which has not been considered. Its behavior remains similar to that of a single-phase flow since the density is not considered in the computation of the spectra. Vapor scalar spectra in the gas phase have been analyzed. A specific procedure has been developed to extract the gas phase information. This procedure has first been validated qualitatively with a dispersed droplet case $(\bar{\phi}=5 \%)$ where the vapor spectrum shows a similar behavior to scalar spectra in single-phase flows. The evaporation process does not seem to affect the spectrum shape but it has a direct influence on the energy level.

\section{Acknowledgement}

This work was granted access to the HPC resources of IDRIS under the allocation 2010x2010026153 made by GENCI (Grand Equipement National de Calcul Intensif). 


\section{References}

Borghi, R., 1988. Turbulent combustion modeling. Prog. Energy Combust. Sci. 14, 245-292.

Brackbill, J. U., Kothe, D. B., Zemach, C., 1992. A continuum method for modeling surface tension. Journal of Computational Physics 100 (2), 335 - 354.

Calimez, X., 1998. Simulation à petite échelle par une méthode v.o.f. d'écoulements diphasiques réactifs. Ph.D. thesis, Ecole Centrale Paris, EM2C.

Chaisemartin, S. D., Fréret, L., Kah, D., Laurent, F., Fox, R., Reveillon, J., Massot, M., 2009. Eulerian models for turbulent spray combustion with polydispersity and droplet crossing. Comptes Rendus Mécanique 337 (6-7), 438 $-448,<$ ce:title $>$ Combustion for aerospace propulsion $</$ ce:title $>$.

Danaila, L., Antonia, R. A., 2009. Spectrum of a passive scalar in moderate reynolds number homogeneous isotropic turbulence. Physics of Fluids 21 (11), 111702.

Demoulin, F. X., Borghi, R., 2002. Modeling of turbulent spray combustion with application to diesel like experiment. Combustion and Flame 129 (3), 281 - 293.

Desjardins, O., Moureau, V., Pitsch, H., 2008. An accurate conservative level set/ghost fluid method for simulating turbulent atomization. Journal of Computational Physics 227 (18), 8395 - 8416.

Enright, D., Fedkiw, R., Ferziger, J., Mitchell, I., 2002. A hybrid particle level set method for improved interface capturing. Journal of Computational Physics 183 (1), 83 - 116.

Eswaran, V., Pope, S., 1988a. Examination of forcing in direct numerical simulations of turbulence. Computers and Fluids 16 (3), $257-278$.

Eswaran, V., Pope, S. B., 1988b. Direct numerical simulations of the turbulent mixing of a passive scalar. Physics of Fluids 31 (3), 506-520.

Fedkiw, R. P., Aslam, T., Merriman, B., Osher, S., 1999. A non-oscillatory eulerian approach to interfaces in multimaterial flows (the ghost fluid method). Journal of Computational Physics 152 (2), 457 - 492.

Gibou, F., Chen, L., Nguyen, D., Banerjee, S., 2007. A level set based sharp interface method for the multiphase incompressible navier-stokes equations with phase change. Journal of Computational Physics 222 (2), 536 - 555.

Guichard, C., Reveillon, J., Haugel, R., 2004. DNS of statistically stationary one-and two-phase turbulent combustion: a turbulent injection procedure,. Turb. Flow \& Comb. 73, 133-167.

Hirt, C. W., Nichols, B. D., 1981. Volume of fluid (vof) method for the dynamics of free boundaries. Journal of Computational Physics 39 (1), $201-225$.

Juric, D., Tryggvason, G., 1998. Computations of boiling flows. International Journal of Multiphase Flow 24 (3), 387 -410 .

Kang, M., Fedkiw, R. P., Liu, X., 2000. Boundary conditions capturing method for multiphase incompressible flow. Journal of Scientific Computing 3, 323-360.

Lebas, R., Menard, T., Beau, P., Berlemont, A., Demoulin, F., 2009. Numerical simulation of primary break-up and 
atomization: Dns and modelling study. International Journal of Multiphase Flow 35 (3), 247 - 260.

Libby, P., Williams, F., 1994. Turbulent reacting flows. Academic Press, London.

Liu, X.-D., Fedkiw, R. P., Kang, M., 2000. A boundary condition capturing method for poisson's equation on irregular domains. Journal of Computational Physics 160 (1), 151 - 178.

Luret, G., Blokkeel, G., Lebas, R., Ménard, T., Berlemont, A., Demoulin, F., 2008. Spray interactions: modeling of collision/coalescence phenomena. In: 22nd European Conference on Liquid Atomization and Spray Systems. Como Lake, Italy.

Luret, G., Menard, T., Blokkeel, G., Berlemont, A., Réveillon, J., Demoulin, F., 2010. Modeling collision outcome in moderately dense spray. Journal of Atomization and Spray 20 (3), 93.

Ménard, T., Tanguy, S., Berlemont, A., 2007. Coupling level set/vof/ghost fluid methods: Validation and application to $3 \mathrm{~d}$ simulation of the primary break-up of a liquid jet. International Journal of Multiphase Flow 33 (5), 510 - 524.

Olsson, E., Kreiss, G., 2005. A conservative level set method for two phase flow. Journal of Computational Physics $210(1), 225-246$.

Osher, S., Fedkiw, R. P., 2001. Level set methods: An overview and some recent results. Journal of Computational Physics 169 (2), $463-502$.

Reveillon, J., Demoulin, F.-X., 2007. Effects of the preferential segregation of droplets on evaporation and turbulent mixing. Journal of Fluid Mechanics 583 (-1), 273-302.

Reveillon, J., Vervisch, L., 2000. Spray vaporization in nonpremixed turbulent combustion modeling: a single droplet model. Combustion and Flame 121 (1-2), 75 - 90.

Reveillon, J., Vervisch, L., 2005. Analysis of weakly turbulent dilute-spray flames and spray combustion regimes. Journal of Fluid Mechanics 537, 317-347.

Rosales, C., Meneveau, C., 2005. Linear forcing in numerical simulations of isotropic turbulence: Physical space implementations and convergence properties. Physics of Fluids 17 (9), 095106.

Schlottke, J., Weigand, B., 2008. Direct numerical simulation of evaporating droplets. Journal of Computational Physics 227 (10), $5215-5237$.

Sethian, J. A., 1996. A fast marching level set method for monotonically advancing fronts. Proceedings of the National Academy of Sciences USA 93 4, 1591.

Shinjo, J., Umemura, A., 2010. Simulation of liquid jet primary breakup: Dynamics of ligament and droplet formation. International Journal of Multiphase Flow 36 (7), 513 - 532.

Shu, C.-W., November 1997. Essentially non-oscillatory and weighted essentially non-oscillatory schemes for hyperbolic conservation laws. Tech. Rep. NASA CR-97-206253 ICASE Report No. 97-65, Institute for Computer Applications in Science and Engineering.

Strotos, G., Gavaises, M., Theodorakakos, A., Bergeles, G., 2008. Numerical investigation on the evaporation of droplets depositing on heated surfaces at low weber numbers. International Journal of Heat and Mass Transfer 
$51(7-8), 1516-1529$.

Sussman, M., Fatemi, E., Smereka, P., Osher, S., 1998. An improved level set method for incompressible two-phase flows. Computers \& Fluids 27 (5-6), $663-680$.

Sussman, M., Puckett, E. G., 2000. A coupled level set and volume-of-fluid method for computing 3d and axisymmetric incompressible two-phase flows. Journal of Computational Physics 162 (2), 301 - 337.

Sussman, M., Smereka, P., Osher, S., 1994. A level set approach for computing solutions to incompressible two-phase flow. Journal of Computational Physics 114 (1), 146 - 159.

Tanguy, S., Berlemont, A., 2005. Application of a level set method for simulation of droplet collisions. International Journal of Multiphase Flow 31 (9), 1015 - 1035.

Tanguy, S., Ménard, T., Berlemont, A., 2007. A level set method for vaporizing two-phase flows. Journal of Computational Physics 221 (2), $837-853$.

Tomar, G., Biswas, G., Sharma, A., Agrawal, A., 2005. Numerical simulation of bubble growth in film boiling using a coupled level-set and volume-of-fluid method. Physics of Fluids 17 (11), 112103.

Tryggvason, G., Esmaeeli, A., Al-Rawahi, N., 2005. Direct numerical simulations of flows with phase change. Computers \& Structures 83 (6-7), 445 - 453, frontier of Multi-Phase Flow Analysis and Fluid-Structure.

Tryggvason, G., Thomas, S., Lu, J., Aboulhasanzadeh, B., 2010. Multiscale issues in dns of multiphase flows. Acta Mathematica Scientia 30 (2), $551-562$.

Unverdi, S. O., Tryggvason, G., 1992. Computations of multi-fluid flows. Physica D: Nonlinear Phenomena 60 (1-4), $70-83$.

Welch, S. W. J., 1995. Local simulation of two-phase flows including interface tracking with mass transfer. Journal of Computational Physics 121 (1), 142 - 154. 


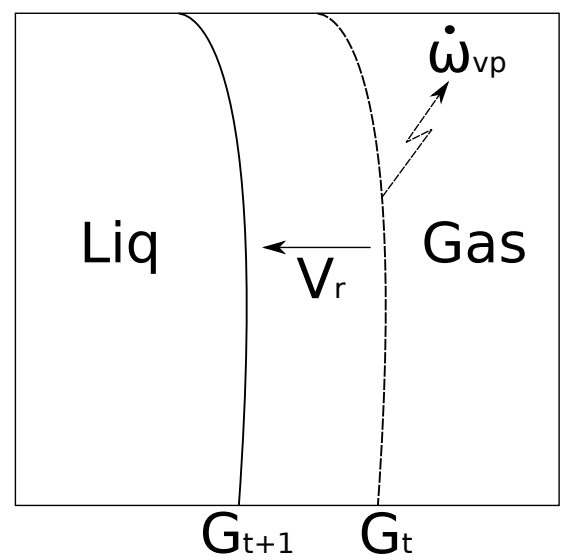

Figure 1: Phase change phenomena at the interface. $V_{r}$ is the regressing velocity, $\dot{\omega}_{v p}$ is the local vaporization rate, $\mathrm{G}$ is the Level Set function.

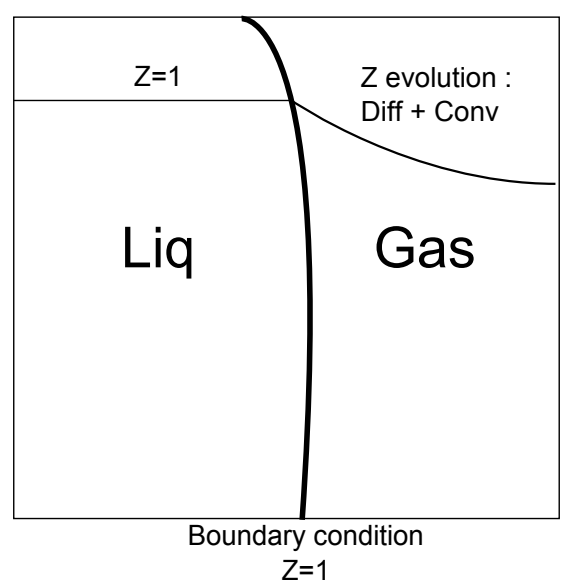

Figure 2: Phase change modeling at the interface in the present work.

Table 1: Parameters of the simulation (S.I. units)

\begin{tabular}{|c|c|c|c|c|c|c|}
\hline$\rho_{l} / \rho_{g}$ & $\sigma$ & $\mu_{g}$ & $\mu_{l}$ & $k_{c}$ & $L$ & $\bar{\phi}$ \\
\hline 30 & 0.0135 & $1.879 .10^{-5}$ & $5.65 .10^{-4}$ & 3.6 & $1.5 .10^{-4}$ & $1-5-10-50-90-95 \%$ \\
\hline
\end{tabular}




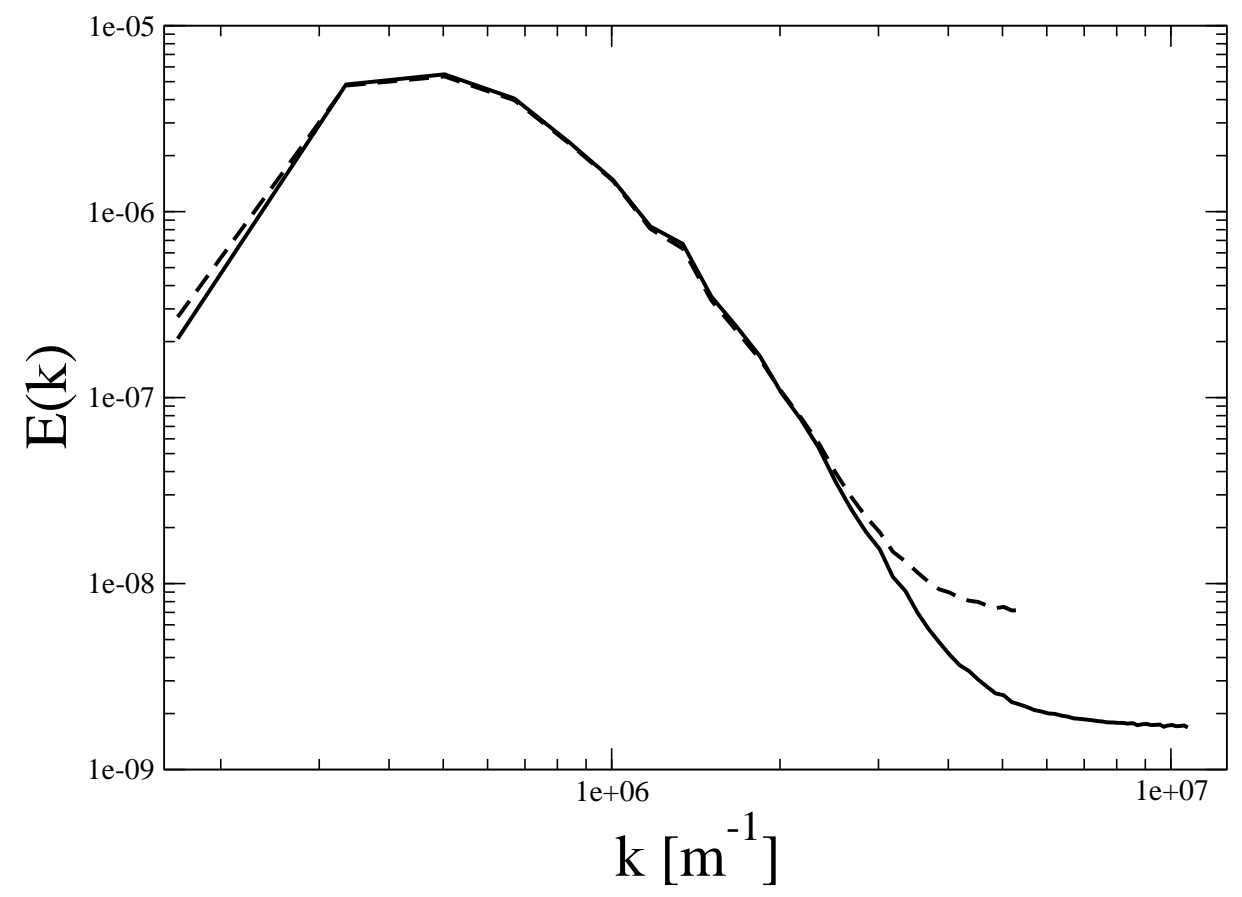

Figure 3: Velocity spectra for two grid resolutions. Dotted line: $128^{3}$ mesh,solid line: $256^{3}$ mesh. 

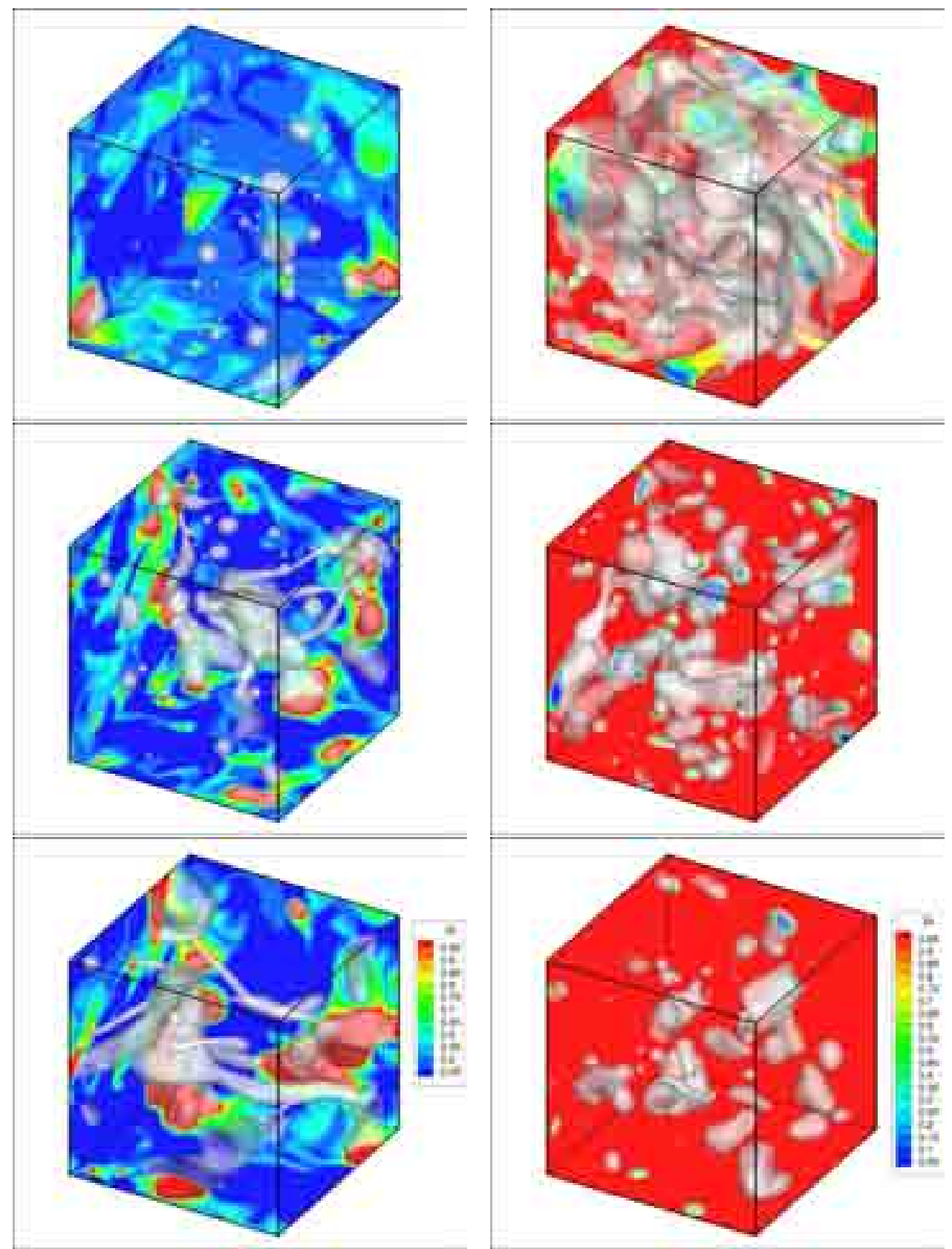

Figure 4: Instantaneous scalar field with interface visualization (in white) at $\bar{Z}=0.5$, for various liquid volume fractions $\bar{\phi}$. The first column represents the scalar field for low $\bar{\phi}(1,5,10 \%$ from top to bottom), and the second column at high $\bar{\phi}(50,90,95 \%$ from top to bottom). 


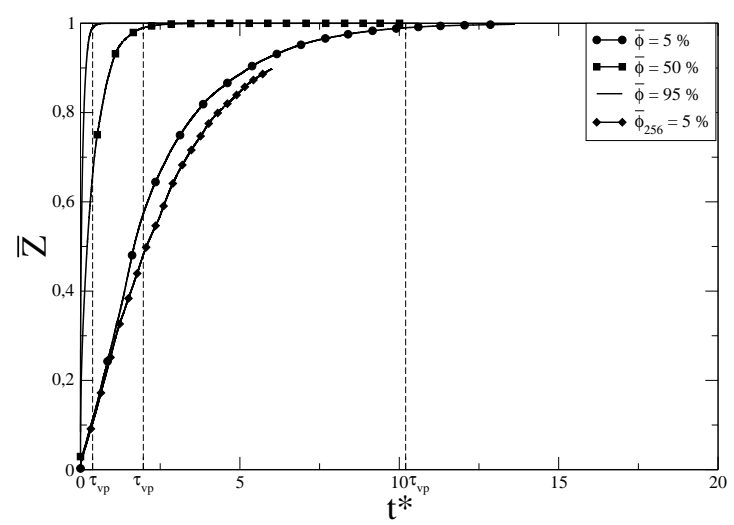

Figure 5: Temporal evolution of the normalized mean vapor concentration for different liquid volume fractions $\bar{\phi}$. 

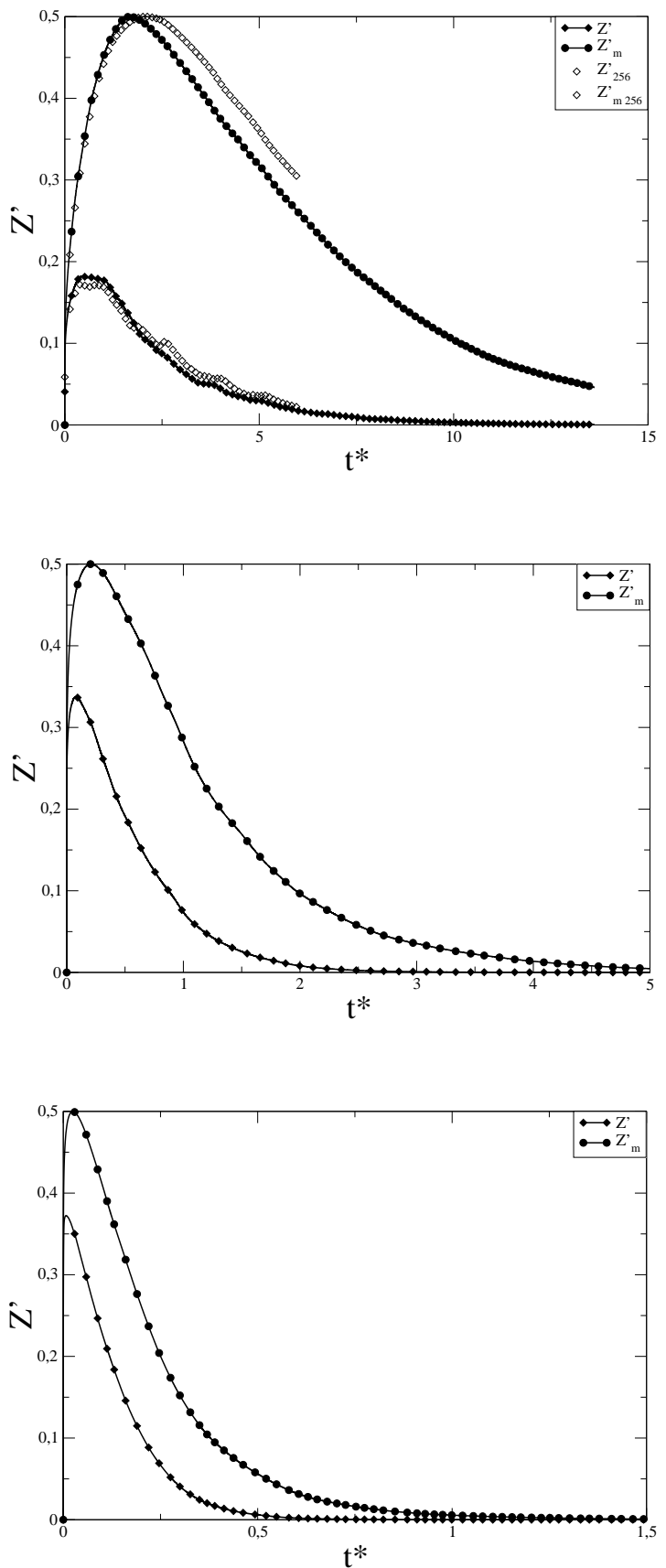

Figure 6: Scalar RMS and max RMS, for $\bar{\phi}=5 \%$ (top), $\bar{\phi}=50 \%$ (middle), $\bar{\phi}=95 \%$ (bottom) 


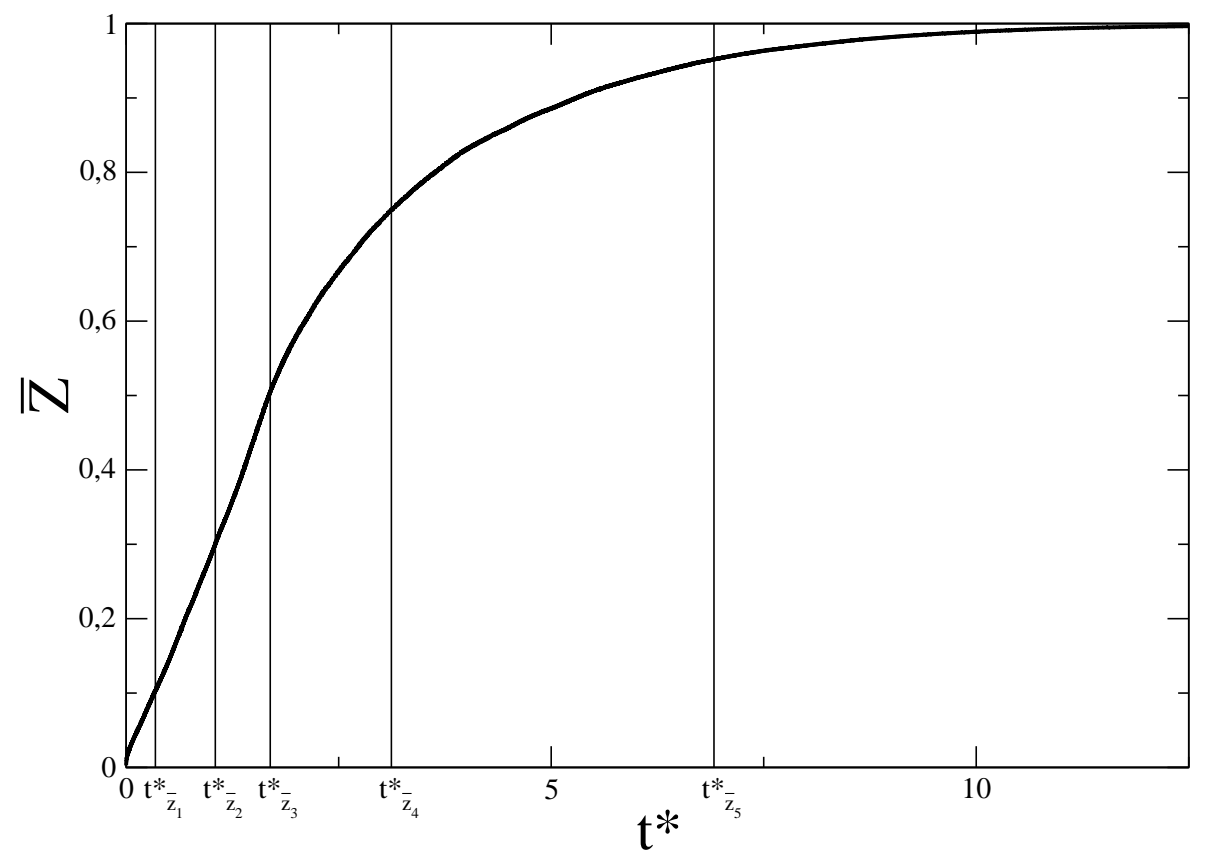

Figure 7: Considered evaporation phases, Scalar mean $\bar{Z}$ for $\bar{\phi}=5 \%$ 

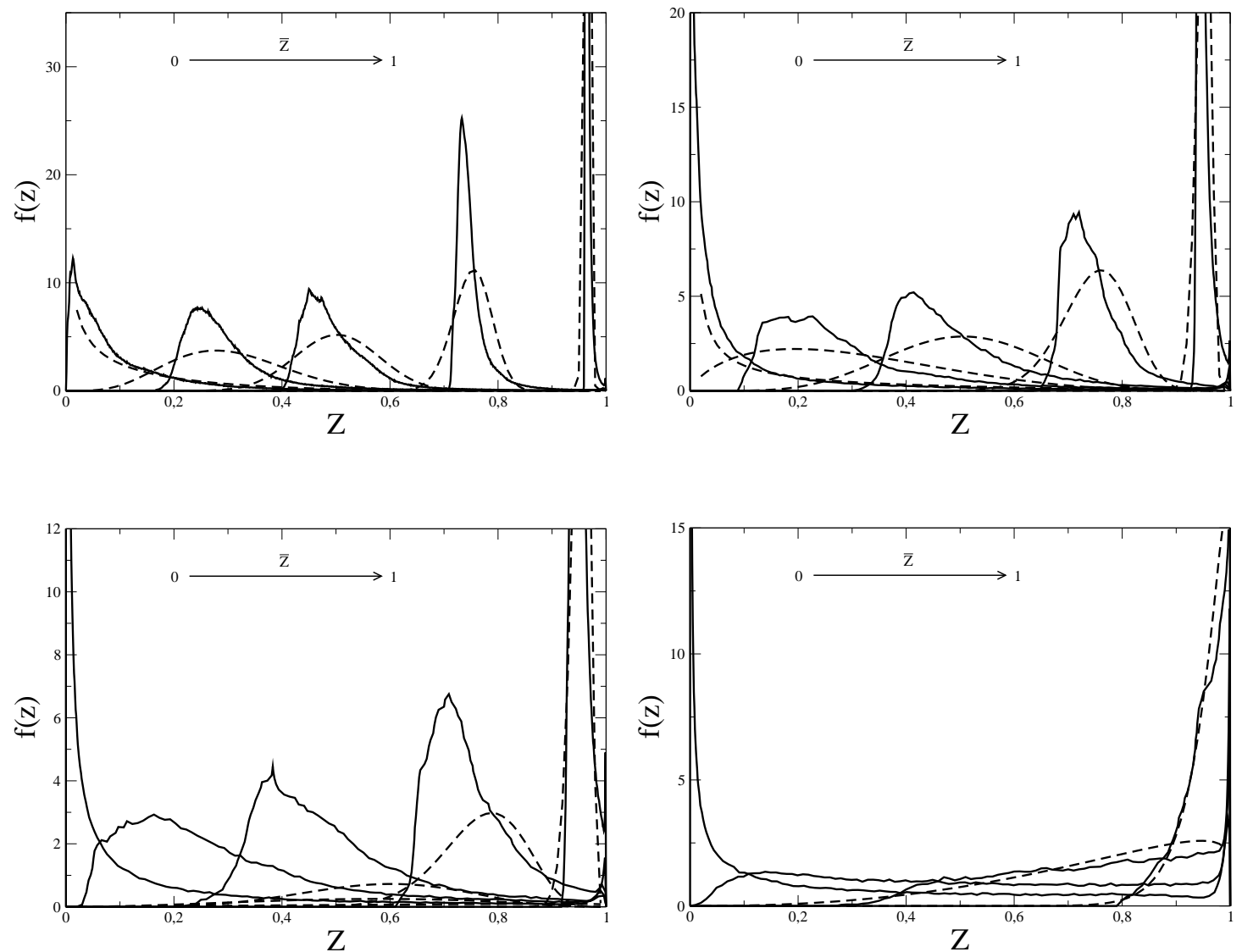

Figure 8: Instantaneous computed PDF (solid line) and beta PDF approximation (dashed line) with the same mean and RMS for $\bar{\phi}=1 \%, 5 \%, 10 \%, 50 \%$ (from left to right, and top to bottom) for the successive phases of the vaporization process, according to Figure 7.

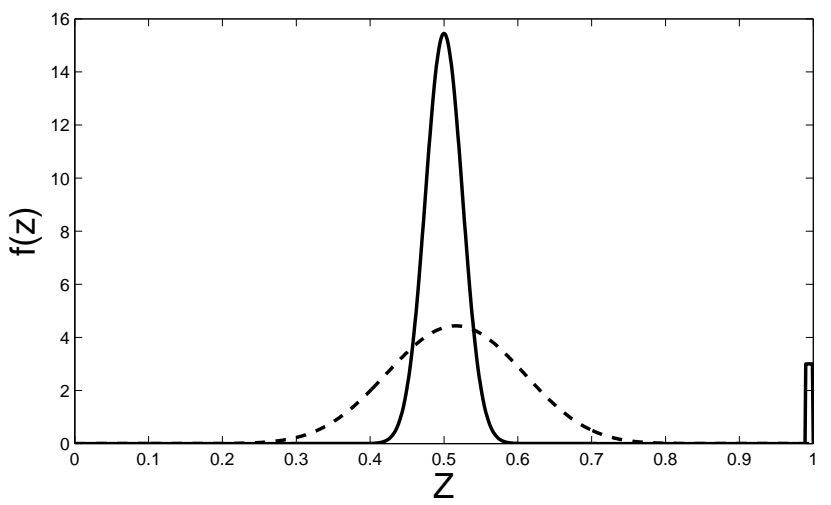

Figure 9: Beta function incompatibility. 

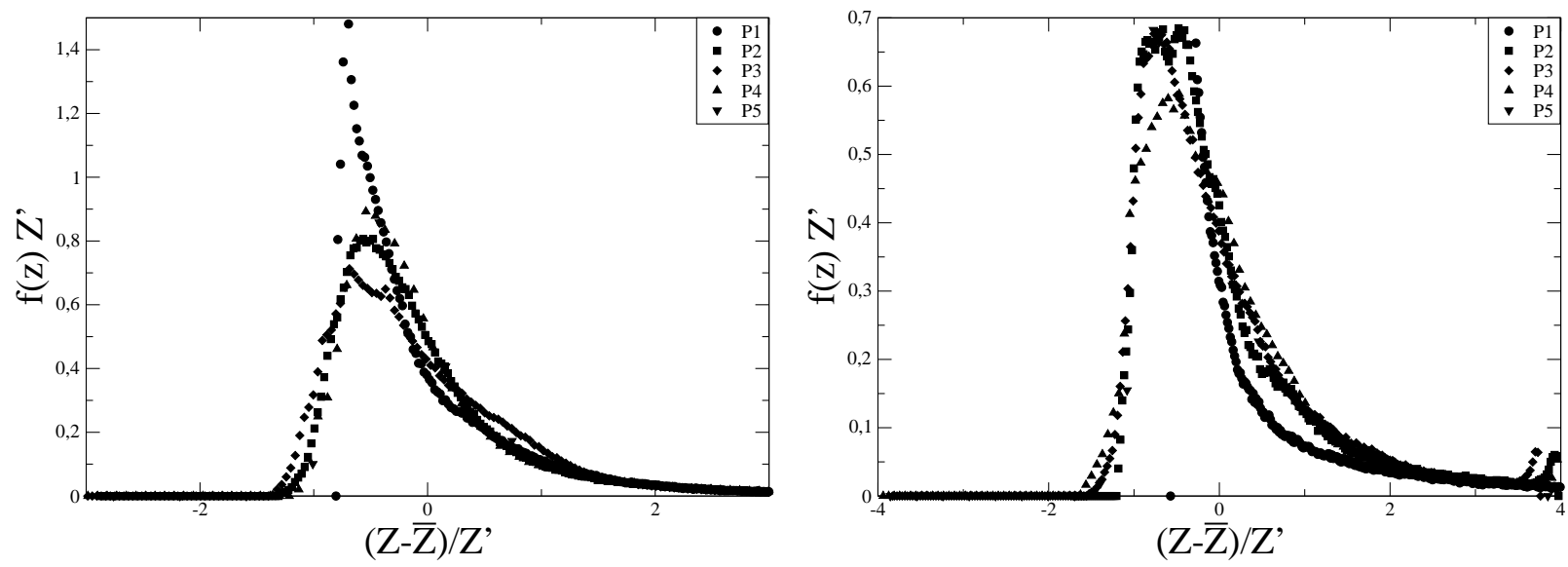

Figure 10: Normalized scalar PDF for $\bar{\phi}=1 \%, 5 \%$ for each phase of the vaporization process.

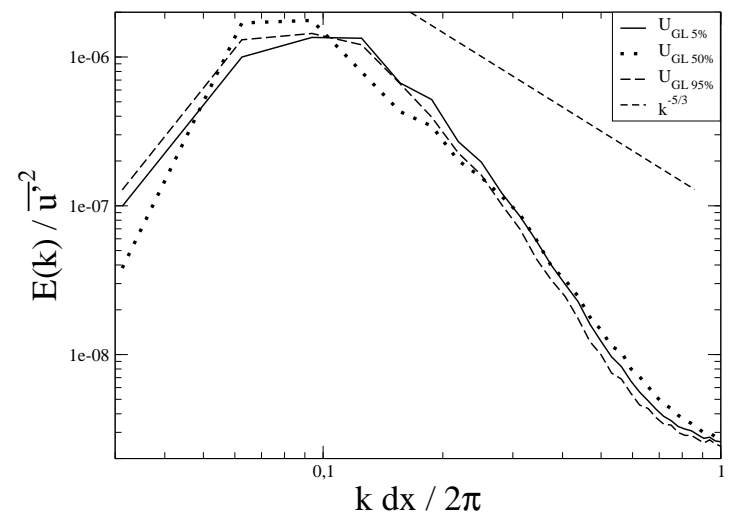

Figure 11: Two-phase velocity spectra for three different liquid volume fractions: $\bar{\phi}=5,50,95 \%$

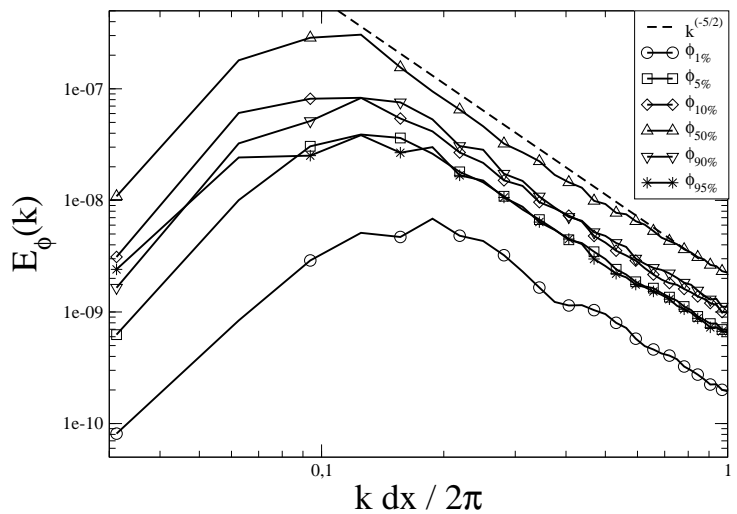

Figure 12: $\phi$ fluctuations spectra for various liquid volume fractions. 

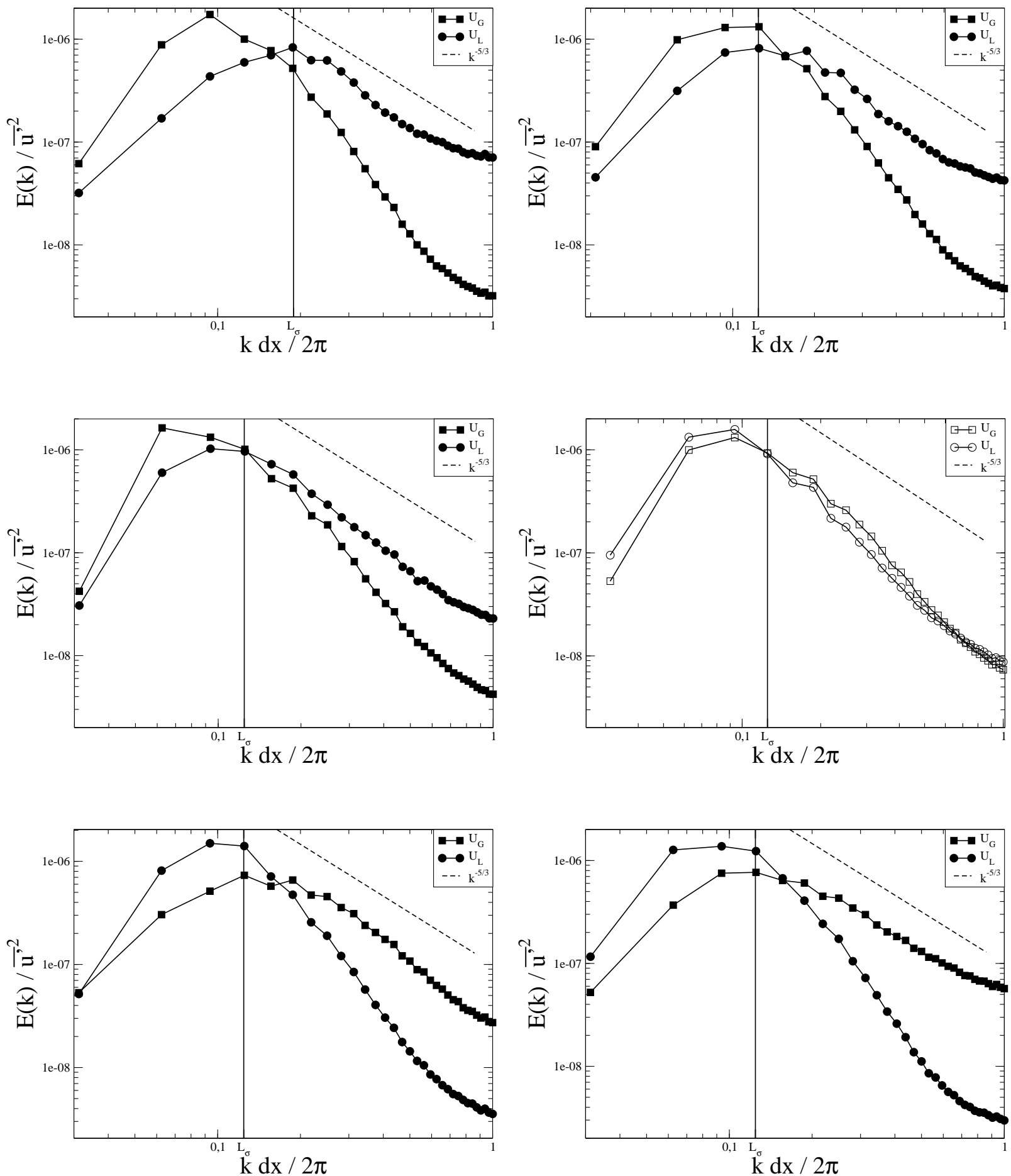

Figure 13: Non-dimensional segregated velocity spectra for $\bar{\phi}=1 \%, 5 \%, 10 \%, 50 \%, 90 \%, 95 \%$ (from left to right, and top to bottom). 


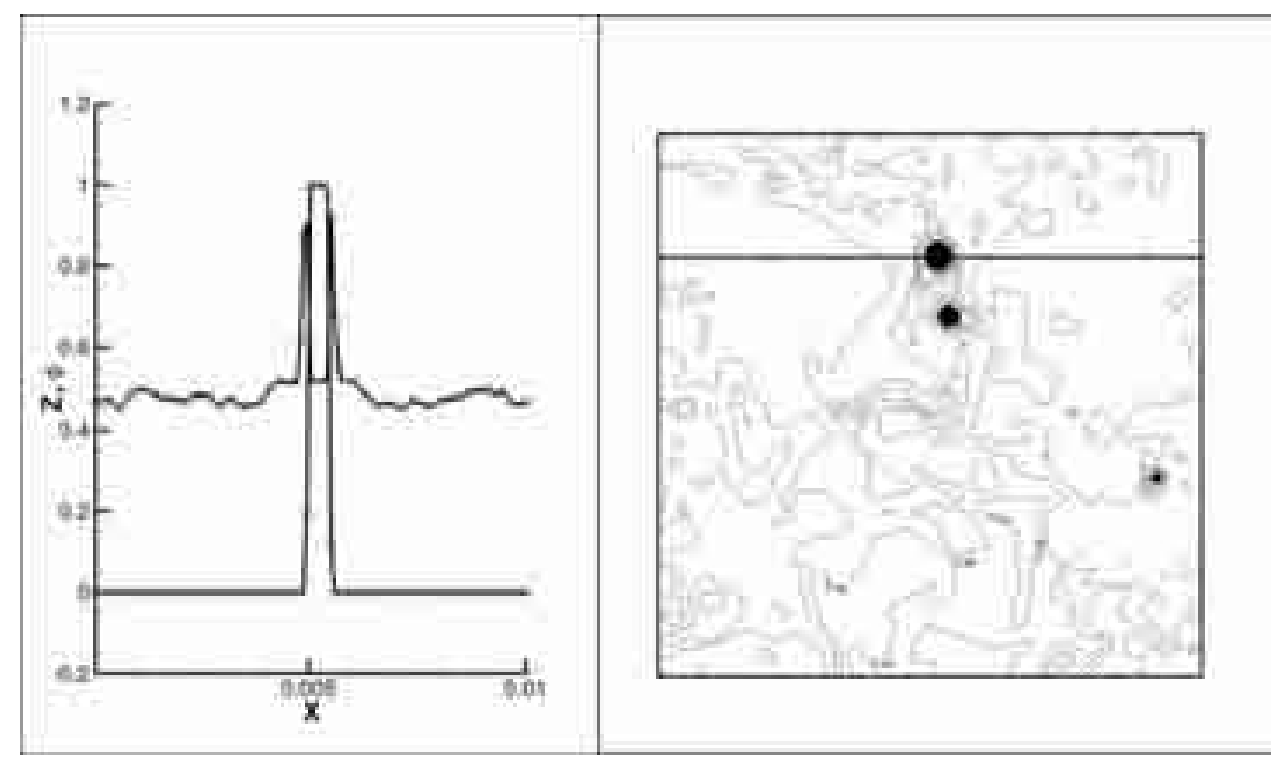

Figure 14: Left: $\mathrm{Z}$ and $\phi$ values over $\mathrm{X}$ axis, square $: \phi$, delta : Z. Right: Scalar iso-contour in thin lines, liquid phase representation in black, $\mathrm{Z}$ values extraction line in bold black.

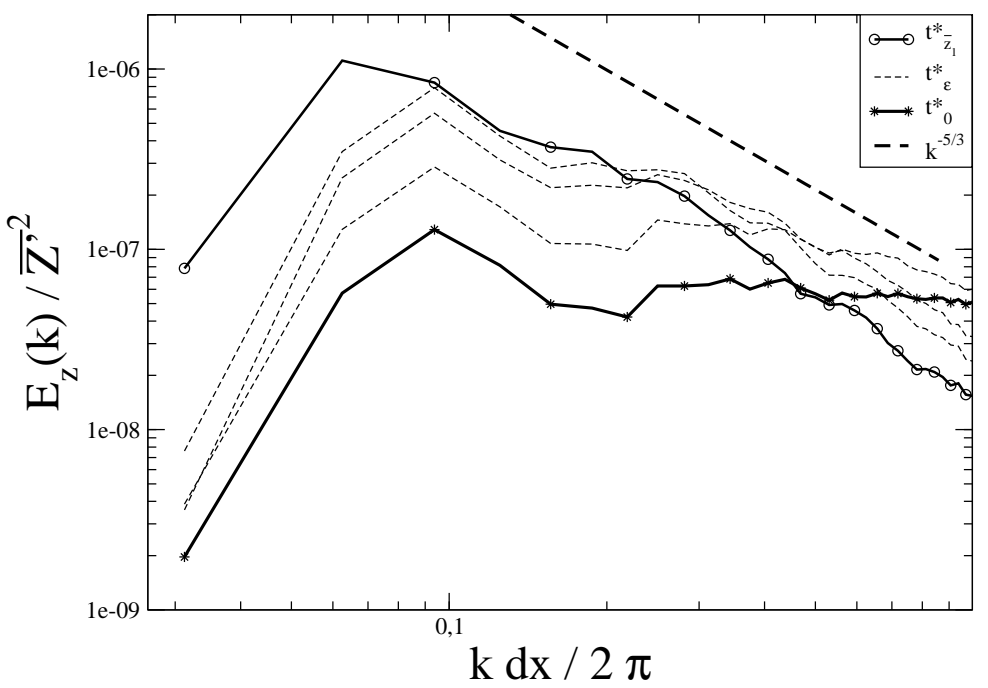

Figure 15: Non-dimensional temporal evolution of Z spectra, for $\bar{\phi}=5 \%$. $t_{0}^{*}$ is the time representing the initial scalar field, $t_{\epsilon}^{*}$ represents short times: $t^{*} \epsilon\left[t_{0}^{*}, t_{\bar{z}_{1}}^{*}\right] . t_{\bar{z}_{1}}^{*}$ is the time corresponding to the first phase of the evaporation process $\left(\bar{Z}_{1}\right)$. 


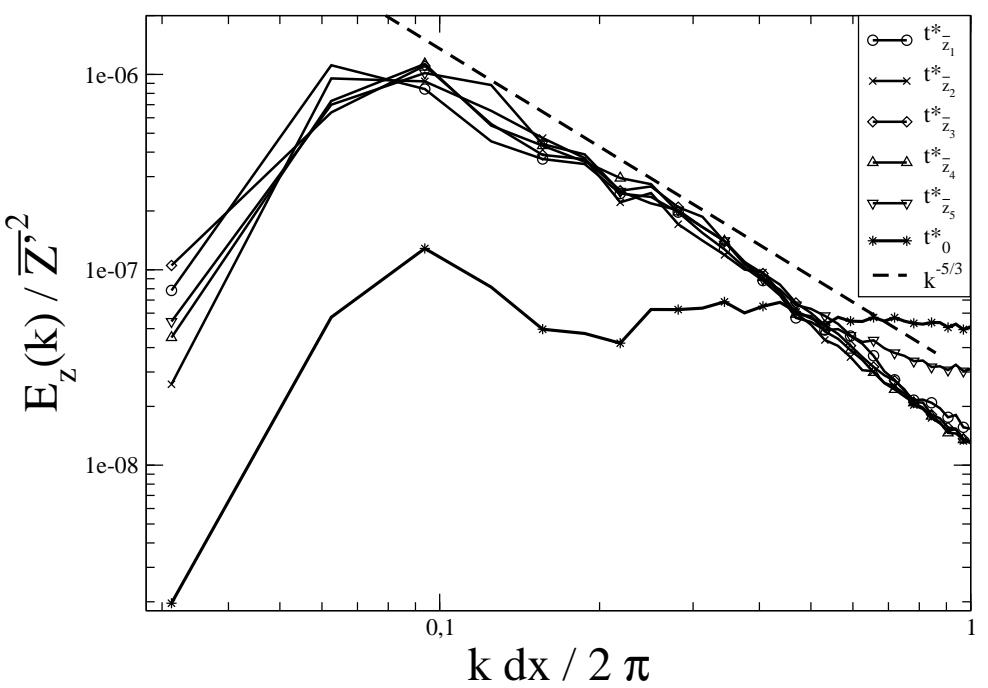

Figure 16: Non-dimensional temporal evolution of $\mathrm{Z}$ spectra for each phase of the vaporization process, $\bar{\phi}=5 \%$. Notations are those used in Figure 6 . 